\section{A forgotten life-threatening medical emergency: myxedema coma}

\author{
Elisa Pizzolato, ${ }^{1,2}$ Alberto Peano, ${ }^{3}$ \\ Letizia Barutta, ${ }^{1,2}$ Emanuele Bernardi, ${ }^{4}$ \\ Elena Maggio, ${ }^{1}$ Bartolomeo Lorenzati, \\ Elena Migliore, ${ }^{1}$ Attilio Allione, ${ }^{1}$ \\ Bruno Maria Tartaglino ${ }^{1}$
}

'Emergency Department, Santa Croce and Carle Hospital, Cuneo; ${ }^{2}$ Emergency Medicine School, University of Turin, Turin; ${ }^{3}$ Medicine School, University of Turin, Turin; ${ }^{4}$ Emergency Department, SS. Annunziata Hospital, Savigliano (CN), Italy

\section{Abstract}

Nowadays myxedema coma is a rare medical emergency but, sometimes, it still remains a fatal condition even if appropriate therapy is soon administered. Although physical presentation is very non-specific and diversified, physicians should pay attention when patients present with low body temperature and alteration of neurological status; the presence of precipitating events in past medical history can help in making a diagnosis. Here we discuss one such case: an 83-year-old female presented with abdominal pain since few days. Laboratory tests and abdomen computed tomography scan demonstrated alithiasic cholecystitis; she was properly treated but, during the Emergency Department stay she experienced a cardiac arrest. Physicians immediately started advance cardiovascular life support algorithm and she survived. Later on, she was admitted to the Intensive Care Unit where doctors discovered she was affected by severe hypothyroidism. Straightway they started the right therapy but, unfortunately, the patient died in a few hours.

\section{Introduction}

Myxedema coma is an uncommon lifethreatening endocrinological emergency: it can occur in patients with a poorly longstanding hypothyroidism or, very rarely, it can represent the first clinical manifestation of an unknown hypothyroidism. Patients affected by hypothyroidism develop many physiological strategies to compensate thyroid hormone lack; when these homeostatic mechanisms fail, patients may move towards mixedematous state. The physical presentation is nonspecific and diversified: diagnosis is easier if a thyroid disease is already known, but it could be a challenge in case of unknown disease. Mortality rate ranges from 30 to $60 \%$ even in case of early detection and appropriate therapy administration.

\section{Case Report}

An 83-year-old woman came to our observation in Santa Croce and Carle Emergency Department (ED) (Cuneo, Italy) complaining of abdominal pain since few days, which was getting worse in the last four hours. She lived alone and she was in good general conditions: she was affected by arthrosis and primary hypertension treated with celecoxib (cyclooxygenase-2 inhibitor) and lercanidipine (calcium channel blocker) respectively. She did not report any other medical disease or medications. At the first clinical examination her vital signs were normal and the electrocardiogram proved atrial fibrillation. She was alert but objectively suffering, with dry, pale, cold and speckled skin. She referred abdominal pain mostly in the right side without signs of peritoneal irritation; ultrasound examination (exploring lungs, aorta, inferior cava vein, abdomen and deep veins) was immediately performed and it did not show any sonographic abnormalities. Laboratory tests demonstrated mildly elevated white blood cells with neutrophilic leukocytosis, normocytic normochromic anaemia, hepatic disfunction with slight elevation of cytolysis and cholestasis indexes, a probable acute kidney failure, mild hyponatremia and hyperkalemia, minimum PT-INR elongation, elevated creatine kinase (CPK) values, high glucose detection and very high c-reactive protein (CRP) levels. Cardiomegaly with minimal pleural effusion and some intestinal fluid levels were respectively detected at chest and abdomen radiographies; the abdomen CT scan showed alithiasic cholecystitis. Endovenous rehydratation, antibiotic therapy (ceftriaxone and metronidazole) and analgesic treatment with morphine were started. Blood pressure decreased in a few minutes and the patient became drowsy: suspecting incipient septic shock, norepinephrine was started. Despite this, the patient lost consciousness developing cardiac arrest; physicians started cardiopulmonary resuscitation and declared Return of Spontaneous Circulation (ROSC) after 10 min. Given the previous detection of acute cholecystitis, patient immediately underwent a diagnostic laparoscopy; surprisingly, the gallbladder walls resulted edematous without any sign of acute cholecystitis. Moreover, no sign of intestinal ischemia was found. Acute myocardial infarction, valvolar dysfunctions, pulmonary embolism and cerebral hemorrhage or
Correspondence: Elisa Pizzolato, Emergency Department, Santa Croce and Carle Hospital, via Michele Coppino 26, 12100 Cuneo, Italy. Tel: +39.0171.642631 - Fax: +39.0171.642635.

E-mail: eli.pizzolato@gmail.com

Key words: Severe hypothyroidism; Myxedema coma; Hypothermia.

Contributions: all authors contributed equally.

Conflict of interest: the authors declare no potential conflict of interest.

Received for publication: 1 February 2016. Revision received: 4 July 2016

Accepted for publication: 4 July 2016

This work is licensed under a Creative Commons Attribution 4.0 License (by-nc 4.0).

CCopyright E. Pizzolato, et al., 2016 Licensee PAGEPress, Italy

Emergency Care Journal 2016; 12:5790

doi:10.4081/ecj.2016.5790

ischemia were excluded on ECG, echocardiography and CT scans respectively. Once admitted to ICU, she presented hypotermic, with low blood pressure and very low heart rate; invasive mechanical ventilation, saline solution (2000 mL/die) and norepinephrine (0.10-0.15 $\mathrm{mcg} / \mathrm{kg} / \mathrm{min}$ ) administration were continued, passive external warming measures and endovenous hydrocortisone (100 $\mathrm{mg}$ tid) were started, but clinical conditions worsened. Blood exams showed increasing hyperkalemia and renal impairment persistence. On past laboratory test revision, a severe hypothyroidism was discovered, probably caused by an immunogenic mechanism [thyroid stimulating hormone (TSH) $93.4 \mu \mathrm{UmL}$, n.l. 0.35-4.7 $\mu \mathrm{UlmL}$, free thyroxine fT4 $3.5 \mathrm{pg} \mathrm{mL}$, n.l. 8-17.6 pglmL, thyroid peroxidase antibodies 930 UlmL, n.l. $<60$ UlmL]. This clinical situation was unknown to our patient since laboratory tests had just been made the day before ED admission. A diagnosis of myxedema coma was formulated. The endocrinologist suggested immediate thyroxine endovenous administration (thyroxine $100 \mathrm{mcg}$ ) and the nephrologist indicated a continuos veno-venous hemodialysis (CVVHD). Unfortunately, after few hours, the patient developed another cardiac arrest and died. Her niece confirmed us the patient complained fatigue since few days so that her family doctor had prescribed blood test to evaluate thyroid status.

\section{Discussion}

Myxedema coma is defined as severe 
hypothyroidism leading to slowing of function in multiple organs. Case series and case reports from western world showed that the incidence of myxedema coma is 0.22 million per year; ${ }^{1}$ in another study including 200 cases between 1953 and 1996, Werner and colleagues demonstrated that when only comatose patients are considered myxedema coma is outermost rare. ${ }^{2}$ Even if diagnosis is now easier, as a result of the widespread availability of TSH assays, mortality rate remains very high (from 30 to 60 percent) despite the best possible treatment. ${ }^{3}$ Hypothyroidism is four time more common in women than in men; myxedema coma occurs quite exclusively in over 60 years people and it tipically occurs during winter months. Myxedema can result from any of the usual causes of hypothyroidsm: primary hypothyroidism due to chronic autoimmune thyroiditis (which can remain latent because of its insidious course), postsurgical or postablative hypothyroidism, in cases of secondary or tertiary hypothyroidism and, rarely, during lithium or amiodarone therapy. ${ }^{4}$

Precipitating events, such as infections (mostly pneumonia, urinary tract infections and cellulitis), certain medications (specially amiodarone, beta blockers, diuretics, lithium and narcotics) and hypoglycemia are the most frequent causes of decompensation into myxedema coma. They disrupt the frail homeostatic compensatory mechanisms in hypothyroid patient and lead to severe hypothyroidism..$^{5-7}$

Burns, carbon dioxide retention, gastrointestinal bleeding, cerebrovascular accident, congestive cardiac failure, surgery, trauma and discontinuation of thyroid supplements are some of the other leading precipitating factors. ${ }^{8}$

Physical examination should focus on the peculiar characteristics of severe hypothyroidism in which many organ systems and metabolic pathways are slowed down. The hallmarks of myxedema coma are the alteration of neurological status and the presence of hypothermia; hypotension, bradycardia, hyponatremia, hypoglycemia and hypoventilation are often present as well. ${ }^{7,9}$

Physical findings include dry skin, sparse hair, hoarse voice, macroglossia, nonpitting edema (due to abnormal mucina deposit into tissues), goiter, myxedematous face with generalized puffiness, thickened nose and swollen lips, ptosis and periorbital edema (it could hide Grave's disease which may have been treated with radioiodine or surgery). Gastrointestinal findings are tipically abdominal distension, decreased intestinal motility, fecal impaction, myxedema megacolon and paralytic ileus, the latter causing bile stasis (directly toxic to the gladbladder epithelium) which leads to alithiasic cholecistitis. The presence of a skin scar on the neck should attract physician attention suggesting postsurgical hypothyroidism: this is an important feature mostly when patient present with neurological impairment. If patient is alert, neurological examination can prove delayed tendon relaxation and an early altered mental status. ${ }^{8,9}$ Despite the appellation of coma, patients present with coma very rarely; they more often present with apathy and lethargy, neglect, decrease in intellectual functions, confusion and obtundation. ${ }^{10}$ The decrease in metabolic functions causes the decrease of thermogenesis bringing to hypothermia. Physicians should remember that a very low body temperature (usually less than $35.5^{\circ} \mathrm{C}$ ) may not be registered by the automatic thermometers: on the other hand, when a low temperature is found, the thermometer itself should be checked to avoid an incorrect measurement. ${ }^{11}$ The most frequent electrolyte abnormalities are hypoglycemia and hyponatriemia. Hypoglycemia may be caused by the down-regulation of metabolism typical in severe hypothyroidism or, more often, it is correlated with a concomitant adrenal insufficiency due to hypothalamic-pituitary disease. Hyponatriemia is the result of decreased free water clearance due to a renal impairment status or an excess of vasopressin secretion: it is classically associated with low serum osmolality and it can contribute to decrease in mental status. ${ }^{2}$ Hypoventilation and respiratory acidosis result primarily from impaired respiratory central drive with decreased response to hypoxemia and hypercapnia ${ }^{13}$ and, secondly, from respiratory muscle weakness, mechanical obstruction exacerbated by macroglossia myxedematous infiltration in pharynx and sleep apneas. ${ }^{14}$

Cardiovascular abnormalities include diastolic hypertension with reduced cardiac output, peripheral vasoconstriction and central shunting, bradycardia and decreased myocardial contractility; decreased oxygen consumption prevents from heart failure. The electrocardiogram shows low voltages and the chest radiography evidences a large cardiac silhouette. ${ }^{15}$ Diagnosis is difficult because of the rarity of the condition and its insidious onset, but it can be clinically suggested by the presence of known thyroid disease, altered mental status, defective thermoregulation and concomitant precipitating factors. ${ }^{16}$ When myxedema coma is highly suspected, blood tests must be performed in order to evaluate TSH, fT4 and cortisol serum concentrations before thyroid hormone and glucocorticoid therapies are administered. Most of patients presenting with myxedema coma are affected by primary hypothyroidism, with high TSH and low free T4 plasma levels. Physicians should not be surprised in case of normal or low TSH and low free T4: they may be facing secondary hypothyroidism (pituitary dysfunction), tertiary hypothyroidism (hypothalamic disorder) or low T4/low T3 syndrome (euthyroid sick syndrome: thyroid function test are altered as a result of illness unrelated to thyroid). ${ }^{8,9}$

Myxedema coma is a medical emergency: in case of high suspicion, physicians should not hesitate in starting replacement therapy while waiting for laboratory tests. The patient should be admitted to the intensive care unit in order to receive ventilatory support, central venous pressure monitoring, appropriate fluid administration, and aggressive management of precipitating factors. The cornerstone of treatment remains thyroid hormone replacement and steroid supplementation in case of coexisting adrenal insufficiency. The optimal treatment still remains controversial because of the very low incidence of the disease and the absence of RCT's or clinical trials that compare different therapies. ${ }^{11}$

High thyroid hormone doses can bring to precipitating fatal tachycardia or myocardial infarction, while, on the other hand, low doses may be inadequate to improve clinical status. ${ }^{17}$ Treatment with T4 provides a slow and steady onset of the action with relatively low adverse reactions, but it may results less effective because of the impaired hormone conversion from the precursor T4 into the active T3. The use of T3 includes quickly shows clinical benefits, especially on neuropsychiatric symptoms, but it exposes tissues to high thyroid hormone concentrations with more frequent side effects. Furthermore, high T3 plasma values during treatment have been correlated with high mortality rate. ${ }^{9}$ Treatment should be initiated intravenously because of possible gastrointestinal absorption impairment; it should be continued orally as soon as possible. Typically, T4 is administered in a loading dose of 200 to $400 \mathrm{mcg}$ to saturate the body pool followed by a daily dose of $1.6 \mathrm{mcg} / \mathrm{kg}$ thereafter. If the clinical status does not improve within 24 hours, addition of $\mathrm{T} 3$ is recommended. Another therapy scheme consists in dispensing T3 in a dose of 5 to $20 \mathrm{mcg}$, followed by 2.5 to 10 mcg every eight hours, depending upon patient's age and the presence of cardiovascular risk factors. T3 is then discontinued when patient improves and reaches clinical stability, while daily oral T4 is maintained. ${ }^{10}$ Assuming a coexisting hypopituitarism and adrenal insufficiency, empiric intravenous glucocorticoid coverage should be employed (stress dose of hydrocortisone $100 \mathrm{mg}$ tid). ${ }^{18}$

Plasma cortisol levels should be drawn before glucocorticoid therapy and, if not depressed, therapy could be discontinued without tapering. ${ }^{8}$

Previously demonstrated predictors of poor outcome are advanced age, bradycardia, cardiovascular disease, reduced consciousness, persistent hypothermia (the lower the temperature, the worse the prognosis) and Sequential Organ Failure Assessment (SOFA) score. ${ }^{17,19,20}$ 


\section{Conclusions}

Myxedema coma is a potentially lethal condition. During last years, diagnosis became easier with TSH blood test availability, but it often remains unrecognized because it is a rare disease. Physicians have to maintain a high index of suspicion when facing the three following clinical conditions: altered mental status, persistent hypothermia and the presence of potential precipitating events. Unfortunately, mortality rate remains high despite early diagnosis and immediate administration of the proper therapy.

\section{References}

1. Galofré JC, Garcia-Mayor RV. [Densidad de incidencia del coma mixedematoso]. [Article in Spanish]. Endocrinologia 1997;44:103-4.

2. Werner SC, Ingbar SH, Braverman LE, Utiger RD. The thyroid: a fundamental and clinical text. 8th ed. Philadelphia, PA: Lipincott Williams \&Wilkins; 2000.

3. Wartofsky L. Myxedema coma. Endocrinol Metab Clin North Am 2006;35:687-98.
4. Santiago R, Rashkin MC. Lithium toxicity and myxedema coma in an elderly woman. J Emerg Med 1990;8:63-6.

5. Rodríguez I, Fluiters E, Pérez-Méndez LF, et al. Factors associated with mortality of patients with myxoedema coma: prospective study in 11 cases treated in a single institution. J Endocrinol 2004;180:347-50.

6. Hassan S, Ayoub W, Hassan M, Wisgerhof M. Amiodarone-induced myxoedema coma. BMJ Case Rep 2014;2014:202338.

7. Brenta G, Vaisman M, Sgarbi JA, et al. Task force on hypothyroidism of the Latin American Thyroid Society (LATS). Clinical practice guidelines for the management of hypothyroidism. Arq Bras Endocrinol Metabol 2013;57:265-91.

8. Wall CR. Myxedema coma: diagnosis and treatment. Am Fam Physician 2000;62: 2485-90.

9. Mathew V, Misgar RA, Ghosh S, et al. Myxedema coma: a new look into an old crisis. J Thyroid Res 2011;2011:493462.

10. Kwaku MP, Burman KD. Myxedema coma. J Intensive Care Med 2007;22:224-31.

11. Wiersinga WM. Myxedema and coma (severe hypothyroidism). South Dartmouth, MA: MDText.com, Inc.; 2015.

12. Nicoloff JT, LoPresti JS. Myxedema coma. A form of decompensated hypothyroidism. Endocrinol Metab Clin North Am
1993;22:279-90.

13. Zwillich CW, Pierson DJ, Hofeldt FD, et al. Ventilatory control in myxedema and hypothyroidism. New Engl J Med 1975;292: 662-5.

14. Lee $\mathrm{CH}$, Wira CR. Severe angioedema in myxedema coma: a difficult airway in a rare endocrine emergency. Am J Emerg Med 2009;27:1021.

15. Klein I. Thyroid hormone and the cardiovascular system. Am J Med 1990;88:631-7.

16. Nicoloff JT. Thyroid storm and myxedema coma. Med Clin North Am 1985;69:100517.

17. Hylander B, Rosenqvist U. Treatment of myxoedema coma: factors associated with fatal outcome. Acta Endocrinol 1985;108: 65-71.

18. Jonklaas J, Bianco AC, Bauer AJ, et al. Guidelines for the treatment of hypothyroidism: prepared by the American thyroid association task force on thyroid hormone replacement. Thyroid 2014;24:1670-751.

19. Beynon J, Akhtar S, Kearney T. Predictors of outcome in myxoedema coma. Crit Care 2008;12:111-2.

20. Dutta P, Bhansali A, Masoodi SR, et al. Predictors of outcome in myxoedema coma: a study from a tertiary care centre. Crit Care 2008;12:R1. 